\title{
Blood-Brain Barrier Permeation: Molecular Parameters Governing Passive Diffusion
}

\author{
H. Fischer ${ }^{1}$, R. Gottschlich ${ }^{2}$, A. Seelig ${ }^{1}$ \\ ${ }^{1}$ Department of Biophysical Chemistry, Biocenter of the University of Basel, Klingelbergstr, 70, CH-4056 Basel, Switzerland \\ ${ }^{2}$ Medicinal Chemistry Research Laboratories, CNS Department, E. Merck, Frankfurter Strasse 250, 64271 Darmstadt, Germany
}

Received: 7 April 1998/Revised: 25 June 1998

\begin{abstract}
. 53 compounds with clinically established ability to cross or not to cross the blood-brain barrier by passive diffusion were characterized by means of surface activity measurements in terms of three parameters, i.e., the air-water partition coefficient, $K_{a w}$, the critical micelle concentration, $C M C_{D}$, and the cross-sectional area, $A_{D}$. A three-dimensional plot in which the surface area, $A_{D}$, is plotted as a function of $K_{a w}^{-1}$ and $C M C_{D}$ shows essentially three groups of compounds: (i) very hydrophobic compounds with large air-water partition coefficients and large cross-sectional areas, $A_{D}>80 \AA^{2}$ which do not cross the blood-brain barrier, (ii) compounds with lower air-water partition coefficients and an average cross-sectional area, $A_{D} \cong 50 \AA^{2}$ which easily cross the blood-brain barrier, and (iii) hydrophilic compounds with low air-water partition coefficients $\left(A_{D}<50 \AA^{2}\right)$ which cross the blood-brain barrier only if applied at high concentrations. It was shown that the lipid membrane-water partition coefficient, $K_{l w}$, measured previously, can be correlated with the air-water partition coefficient if the additional work against the internal lateral bilayer pressure, $\pi_{b i}=34 \pm 4 \mathrm{mN} / \mathrm{m}$ is taken into account. The partitioning into anisotropic lipid membranes decreases exponentially with increasing cross-sectional areas, $A_{D}$, according to $K_{l w}=$ const. $K_{a w} \exp \left(-A_{D} \pi_{b i} / k T\right)$ where $k T$ is the thermal energy. The cross-sectional area of the molecule oriented at a hydrophilic-hydrophobic interface is thus the main determinant for membrane permeation provided the molecule is surface active and has a $\mathrm{pK}_{a}>4$ for acids and a $\mathrm{pK}_{a}<10$ for bases.
\end{abstract}

Key words: Blood-brain barrier — Passive diffusion Monolayer-bilayer equivalence pressure - Internal lateral bilayer pressure - Molecular size - Cross-sectional area - Amphiphilicity - $\mathrm{pK}_{a}$ - Drug screening

Correspondence to: A. Seelig

\section{Introduction}

Passive diffusion through the blood-brain barrier (BBB) is the primary process of translocation from the blood stream to the brain for the large majority of therapeutic compounds. On a morphological level, the BBB consists of the brain microvascular endothelium cells coupled by tight junctions. On a molecular level, the principal diffusion barrier consists of the lipid bilayer. The latter is a highly anisotropic system which can be divided into at least two distinct regions: (i) the polar head group-water interface region, and (ii) the nonpolar hydrocarbon region. Deuterium nuclear magnetic resonance $\left({ }^{2} \mathrm{H}-\mathrm{NMR}\right)$ (Seelig \& Seelig, 1974; Seelig \& Seelig, 1980) and neutron diffraction measurements (Büldt et al., 1978) have shown that the order of the hydrocarbon chains is relatively high in the region near the lipid-water interface and decreases strongly towards the bilayer center. These results demonstrate that a bilayer cannot be properly mimicked by an isotropic hydrocarbon phase. Indeed, molecular dynamics simulations show that the rate of diffusion differs considerably even in the membrane hydrocarbon region, being slow in the region of high order and fast in the more disordered central part (BassolinoKlimas, Alper \& Stouch, 1993).

Despite the well established anisotropy of lipid bilayers, the vast majority of investigations still relies on the measurement of partition coefficients between an organic solvent (e.g., octanol, hexane or cyclohexane) and water to assess the ability of a drug to diffuse through the $\mathrm{BBB}$. Interesting modifications of this approach are HPLC methods where alkyl chains (e.g., C-18) (Kaliszan, 1990), or carboxyacyl phosphocholine chains (e.g., 11-carboxylundecylphosphocholine) (Ong et al., 1995; Yang et al., 1997) are covalently attached to silica. These systems show a limited anisotropy. However, the packing density is still dictated by the chemistry of the covalent linkage to silica and is distinctly lower than 
the lipid packing density of a natural membrane (Demel et al., 1975) or a bilayer model membrane (Seelig, 1987). It is therefore not surprising that octanol-water partition coefficients or lipophilicity parameters obtained from reversed phase HPLC measurements do not correlate satisfactorily with the ability of a drug to diffuse through a lipid membrane. Unfortunately, measurements of drug binding and permeation through lipid bilayer vesicles are generally time consuming.

As an alternative we have recently suggested a method which does not involve lipids or organic solvents (Seelig, Gottschlich \& Devant, 1994). Our approach is based on the measurement of the Gibbs adsorption isotherm of the molecule of interest. The Gibbs adsorption isotherm is a quantitative measure for the tendency of a drug to move to the air-water interface. The molecular organization of drug molecules is not random but ordered such that the nonpolar parts extend into the air while the hydrophilic parts maximize their contact with the water phase. Since air has a similar dielectric constant as the hydrocarbon region of the lipid membrane, the air-water interface provides a good model for drug orientation at the lipid-water interface. In addition, the analysis of the Gibbs adsorption isotherm yields three physical-chemical parameters of a drug molecule: (i) the minimum concentration to induce surface activity, $C_{0}$, (ii) the surface area of the molecule at the air-water interface, $A_{D}$, and (iii) the critical micelle concentration, $C M C$. These three parameters together with the ionization constants of the drugs allow a separation of compounds which reach the central nervous system (CNS) and compounds which do not reach it with a predictive success of better than $90 \%$ (Seelig et al., 1994).

The aim of the present work is, first, to provide a better physical chemical understanding of the empirical parameters used previously. This is possible by describing the Gibbs adsorption isotherm by means of the Szyszkowski equation ( $c f$. e.g., Rosen, 1989). The latter provides an air-water partition coefficient, $K_{a w}$, which correlates with the concentration of surface activity onset, $C_{0}$. Secondly, we discuss the crucial importance of the surface area requirement of the drug, $A_{D}$, in connection with the bilayer anisotropy. The penetration of a drug between the hydrocarbon chains requires energy which is small for small molecules but can become prohibitively high for molecules with a large cross section. Finally, we extend our previous approach to a much larger list of compounds.

\section{Materials and Methods}

\section{MATERIALS}

53 compounds were selected and classified according to their ability to enter the CNS by passive diffusion through the BBB (Table). Com- pounds identified to easily cross the BBB by passive diffusion are denoted $\mathrm{CNS}^{+}$, those which cannot pass the BBB — or only to a very limited extent - are denoted $\mathrm{CNS}^{-}$in the following.

Compounds which are therapeutically used for CNS indications are considered as $\mathrm{CNS}^{+}$. In addition, the antihypertensive, $\mathrm{mCPP} \cdot 2 \mathrm{HCl}$ which undoubtedly acts by means of a central mechanism (Fuller, 1986) and the analgesic, spiradoline (Von Voightlander \& Lewis, 1988) are also considered at $\mathrm{CNS}^{+}$.

\section{Hydrophobic $\mathrm{CNS}^{-}$COMPOUNDS WITH POTENTIAL Metabolic Modifications}

The characterization of hydrophobic compounds as $\mathrm{CNS}^{-}$is more complex since they may undergo first-pass metabolism. We therefore discuss the potential metabolic modifications of these compounds and the ability of their metabolites to cross the BBB.

Amiodarone undergoes first pass metabolism in the liver. The unmetabolized form was nevertheless observed in different peripheral organs, but not in the brain (Broekhuysen, Laruel \& Sion, 1969). Astemizole is also metabolized to a certain extent but relevant levels of the unchanged substance were still detected in the plasma. No side effects were observed, even at high concentrations (Seppala \& Savolainen, 1982) which suggests that it does not reach the brain (Reece et al., 1994). Asimadoline reaches the brain only to a limited extent as shown by means of in vitro experiments (Barber et al., 1994). Domperidone is a peripherally active dopamine antagonist. It does not induce the central effects generally observed for centrally active dopamine antagonists after intravenous injection (Reyntjens et al., 1978) and can therefore be assumed not to reach the brain. Ebastine undergoes almost complete first pass metabolism to carebastine after oral absorption. Due to the absence of central effects it can be concluded that the latter does not reach the brain. After parenteral injection ebastine is found, at least initially, in high levels in the plasma (Martinez-Tobed et al., 1992; Matsuda et al., 1994); however, no central effects have been observed (Moragues \& Roberts, 1990). Loperamide, a peripherally selective opiate, shows plasma concentrations in the range of $2 \mathrm{ng} / \mathrm{ml}$ after oral absorption in man (Heykants et al., 1974). Nevertheless, no central opioid effects were observed, even at concentrations which distinctly exceeded therapeutic concentrations (Schuermans et al., 1974). Terfenadine undergoes first pass metabolism to fexofenadine, a metabolite containing a carboxylic acid group. However, the unmetabolized form could still be detected in the blood and in a number of organs but not in the brain (Leeson et al.,1982).

\section{P-Glycoprotein Substrates}

Since active efflux of compounds by P-glycoprotein can interfere with passive diffusion we screened the structures of all compounds for potential P-glycoprotein recognition patterns (Seelig, 1998). In brief, substrate recognition patterns for P-glycoproteins have been proposed to consist of two electron donor groups with a spatial separation of 2.5 $\pm 0.3 \AA$ (type I unit), two electron donor groups with a spatial separation of $4.6 \pm 0.6 \AA$ (type II unit) or three electron donor groups with a spatial separation of the outer two groups of $4.6 \pm 0.6 \AA$ (type II unit) and to interact via hydrogen bonding with the transmembrane sequences of P-glycoprotein. The binding capacity of substrates is given in terms of hydrogen bonding energy units (e.u.) in the Table. Only compounds with e.u. $\leqslant 4$ have been selected (Table) in order to minimize the interference of active efflux by P-glycoprotein with passive diffusion.

\section{Measurement of Surface Activity}

Experiments were essentially performed as described previously (Seelig et al., 1994). Water used for buffers and solutions was nano- 
pure with a resistivity of $17.5 \mathrm{M} \Omega \cdot \mathrm{cm} .50 \mathrm{mM}$ Tris buffer containing $114 \mathrm{~mm} \mathrm{NaCl}$ was adjusted with $\mathrm{HCl}$ to the desired $\mathrm{pH}$. Measurements were performed at $23 \pm 1^{\circ} \mathrm{C}$.

Two home-built Teflon troughs ( 20 and $3 \mathrm{ml}$ filling volume) were used. For both troughs the surface pressure was measured with a Wilhelmy plate. The electronically controlled equilibration time of the spring to which the Wilhelmy plate is attached was much shorter for the $3 \mathrm{ml}$ trough. As a consequence the measurement of a Gibbs adsorption isotherm required only about $45 \mathrm{~min}$ in the $3 \mathrm{ml}$ trough but about $4 \mathrm{hr}$ in the $20 \mathrm{ml}$ trough.

To minimize evaporation both troughs were covered by a plexiglas hood. Drug solutions were injected in small increments by means of a microsyringe to achieve the desired concentrations. In the case of the $20 \mathrm{ml}$ trough the added volume (maximum $\leqslant 2 \mathrm{ml}$ ) exactly compensated the loss of solvent via evaporation. For the $3 \mathrm{ml}$ trough, the addition of solvent exceeded the loss of solvent by evaporation because of the shorter measuring time. Concentrations were therefore corrected for solvent addition as well as for evaporation.

For measurements in the $20 \mathrm{ml}$ trough, stock solutions were $10^{-1}$ $10^{-3} \mathrm{M}$. Due to the relatively high concentrations, methanol was used as solvent, independent of the respective water solubility of the compound. Since methanol is itself surface active, the surface pressure, $\pi$, was corrected for the surface pressure of methanol. For measurements in the $3 \mathrm{ml}$ trough the concentrations of the stock solutions were only $10^{-2}-10^{-4} \mathrm{M}$ and water could therefore by used as a solvent. The $\pi / C$ curves measured for compounds dissolved in water corresponded well to the $\pi / C$ curves of compounds dissolved in methanol and corrected for the surface pressure of methanol. The reproducibility of the measurements was $\pm 5 \%$ including preparation of stock solution and buffer solution.

\section{Measurement of the Lipid Membrane-Water PARTITION COEFFICIENT}

Thermodynamics of drug partitioning into lipid bilayers will be discussed in detail elsewhere (A. Frentzel, X. Li, and A. Seelig, in preparation). In brief, the partitioning of drugs into sonified POPC vesicles was measured by means of high sensitivity isothermal titration calorimetry in buffer solution at $28^{\circ} \mathrm{C}$. Using a Hamilton syringe coupled with a stepping motor, $10 \mu \mathrm{l}$ aliquots of the phospholipid vesicle suspension $\left(C_{L}=33 \mu \mathrm{M}\right)$ were injected into the calorimeter cell (volume $V_{\text {cell }}=1.3353 \mathrm{ml}$ ) containing the drug solution. Each injection gave rise to an exothermic heat of reaction, produced by the partitioning of the drug into the membrane (for further details $c f$. Wenk et al., 1997). Asimadoline $\mathrm{HCl}(20 \mu \mathrm{M})$, and astemizole $2 \mathrm{HCl}(10 \mu \mathrm{M})$ were dissolved in $50 \mathrm{mM} \mathrm{KH}_{2} \mathrm{PO}_{4} / \mathrm{K}_{2} \mathrm{H} \mathrm{PO}_{4}$ at $\mathrm{pH} 5.2$ containing $114 \mathrm{~mm} \mathrm{NaCl}$. The low $\mathrm{pH}$ was chosen because of aggregation problems at higher $\mathrm{pH}$. Desipramine $\mathrm{HCl}(50 \mu \mathrm{M})$, chlorpromazine $\mathrm{HCl}(50 \mu \mathrm{M})$, mequitazine $\mathrm{HCl}(50 \mu \mathrm{M})$, perphenazine $\mathrm{HCl}(50 \mu \mathrm{M})$, and cis-flupenthixol $\mathrm{HCl}(10$ $\mu \mathrm{M})$ were dissolved in $50 \mathrm{~mm}$ Tris/ $\mathrm{HCl}$ at $\mathrm{pH} 7.4$ containing $114 \mathrm{~mm}$ $\mathrm{NaCl}$. Concentrations were chosen as low as possible in order to avoid drug association in solution. For the evaluation of the lipid membranewater partition coefficients, $K_{l w}$, regarded as a surface partition coefficient, charge effects were taken into account by means of the GouyChapman theory (cf. McLaughlin, 1989).

\section{Theory}

The adsorption of an amphiphile at the air-water interface lowers the surface tension of the buffer, $\gamma_{0}$, to a new value $\gamma$. The difference, $\pi=\gamma_{0}-\gamma$ is the so-called surface pressure. The thermodynamics of the adsorption process is described by the Gibbs adsorption isotherm which can be written as

$d \gamma=-R T\left(N_{A} A_{S}\right)^{-1} d \ln C=-R T \Gamma d \ln C=-d \pi$

where $C$ is the concentration of the amphiphile in bulk solution, $R T$ is the thermal energy, $N_{A}$ is the Avogadro number and $A_{S}$ is the surface area of the surface active molecule at the interface. $\Gamma=\left(N_{A} A_{S}\right)^{-1}$ is denoted surface excess concentration. At low concentrations $\Gamma$ increases linearly with $C$, at high concentrations $\Gamma$ reaches a limiting value $\Gamma_{\infty}$. A plot of $\pi$ vs. $\ln C$ should yield a straight line as long as $\Gamma$ is constant. $A_{S}$ was evaluated from the slope

$\Gamma_{\infty}=(1 / R T) \mathrm{d} \pi / d \ln C$

Integral forms of Eq. (1) can also be given. Particularly useful for our purpose is the Szyszkowski equation ( $c f$. e.g., Rosen, 1989) which may be written as

$\pi=R T \Gamma_{\infty} \ln \left(1+K_{a w} C\right)$

where $K_{a w}$ is the air-water partition coefficient. By fitting Eq. (3) to the measured $\pi / C$ curve using $\Gamma_{\infty}$, determined according to Eq. (2), the air-water partition coefficient, $K_{a w}$, was evaluated.

\section{Results}

\section{THE $\pi / C$ ISOTHERM}

Figure $1 A$ shows the variation of the surface pressure, $\pi$, with the logarithm of drug concentration, $\mathrm{C}$, for astemizole, a $\mathrm{CNS}^{-}$-compound, at $\mathrm{pH} 7.4$ in buffer. The slope of the linear part of the Gibbs adsorption isotherm (dashed line) yields the surface excess concentration, $\Gamma_{\infty}$, and, in turn, the surface area requirement of astemizole $\left(A_{S}=94 \pm 5 \AA^{2}\right)$. Figure $1 B$ shows the same measurement with the logarithmic scale replaced by a linear scale for the concentration, $\mathrm{C}$. The solid line represents the binding isotherm calculated with the Szyszkowski equation (Eq. 3). The simulation uses the surface area as determined from the Gibbs adsorption isotherm (Fig. 1A) and yields a partition coefficient of $K_{a w}=(31.6 \pm 4.6)$ $10^{4} \mathrm{M}^{-1}$. The deviations at very low drug concentrations are due to adsorption to the surface of the Teflon trough. For more hydrophilic compounds adsorption is less pronounced. Altogether 53 drugs were measured and analyzed by this approach and the data are summarized in the Table.

At drug concentrations, $C=1 / K_{a w}$ the excess surface concentration, $\Gamma$, reaches its half maximum value 

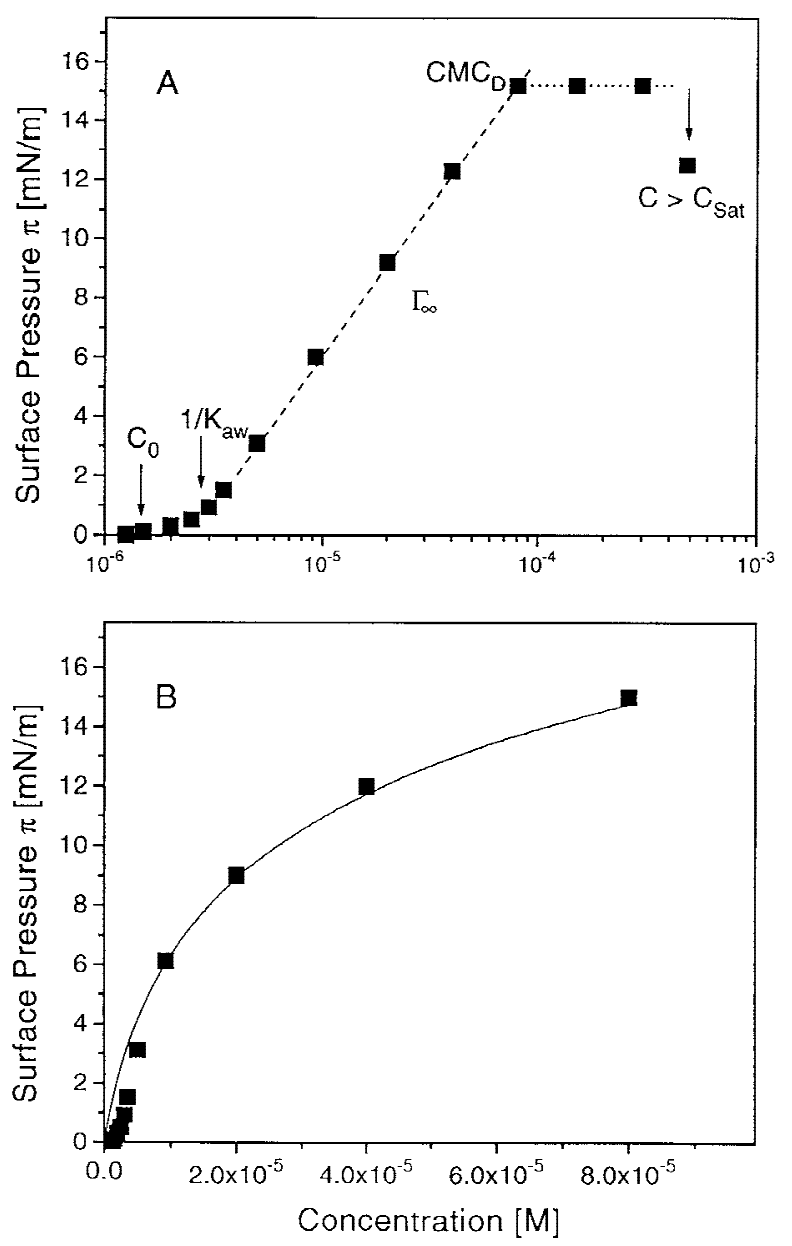

Fig. 1. $A$ The Gibbs adsorption isotherm $(\pi / \log C)$ of astemizole measured in buffer solution at $\mathrm{pH} 7.4(50 \mathrm{~mm}$ Tris/ $\mathrm{HCl}, 114 \mathrm{~mm} \mathrm{NaCl})$. The parameters derived from the Gibbs adsorption isotherm are: (i) the concentration of surface activity onset, $C_{o}$, (ii) the air-water partition coefficient, $K_{a w}$, (iii) the critical micelle concentration, $C M C_{D}$, (iv) the concentration of saturation $C_{s a t}$ and (v) the surface excess concentration, $\Gamma_{\infty}$, from which the surface area of the molecule at the air-water interface, $A_{s}$, can be determined. $B: \pi / C$ - curve of astemizole, the solid line represents the binding isotherm calculated with the Szyszkowski equation (Eq. 3).

$\Gamma_{\infty} / 2$ (cf. Fig. 1A). Previously (Seelig et al., 1994) we introduced an empirical parameter $C_{o}$, which was arbitrarily defined as the drug concentration at which the surface pressure is $0.1 \mathrm{mN} / \mathrm{m}$. The two parameters are strongly correlated ${ }^{1}$. Since $K_{a w}$ is physically better defined we will use this parameter in the following. However, for compounds with low amphiphilicity or amphiphilicity changing in the process of aggregation ( $c f$. be-

\footnotetext{
${ }^{1}$ A plot of $\log C_{o}$ vs. $\log K_{a w}$ yields a linear correlation $\left(\log K_{a w}=\right.$ $\left.-0.96( \pm 0.29)-1.11( \pm 0.06) \log C_{o}\right)$ with a correlation coefficient of 0.971 .
}

low) the measured Gibbs adsorption isotherm cannot be satisfactorily simulated. For these compounds neither $A_{s}$ nor $K_{a w}$ can be properly determined and we therefore still use the $C_{o}$ instead of the $K_{a w}$ values.

As indicated in Fig. $1 A$ two further parameters can be derived from the Gibbs adsorption isotherm: the critical micelle concentration of a drug molecule, $C M C_{D}$, and the concentration of saturation, $C_{\text {sat }}$. At the $C M C_{D}$ the surface pressure, $\pi$, becomes independent of the drug concentration indicating micelle formation. Micelles formed by amphiphilic drugs are generally less stable than micelles formed by detergents (Paula et al., 1995). The $\mathrm{pK}_{a}$ values of drugs tend to shift (to lower values for bases and to higher values for acids) with increasing concentration (Fischer, 1998; Mayer et al., 1988) leading to further aggregation and finally precipitation. This process is experimentally observed as a collapse of surface pressure at the concentration of saturation, $C_{s a t}$. The latter corresponds to about 3-4 times the $C M C_{D}$ for compounds with average amphiphilicity ( $c f$. below), but can almost coincide with the $C M C_{D}$ for compounds with low amphiphilicity.

\section{The Cross-Sectional Area of Drugs}

The large majority of drug molecules investigated here carry charged groups. For charged molecules the surface area, $A_{S}$, depends on the $\mathrm{pH}$ of the solution. As an example the $\mathrm{pH}$ dependence of the surface area of hydroxyzine $\cdot 2 \mathrm{HCl}$ is shown in Fig. 2. Hydroxyzine carries two tertiary amino groups with $\mathrm{pK}_{a, 1}=7.2$ and $\mathrm{pK}_{a, 2}=2.1$ (Newton \& Kluza, 1978). The surface area, $A_{S}$, is large at low $\mathrm{pH}$ due to charge repulsion effects and decreases with increasing $\mathrm{pH}$ in a sigmoidal manner. The inflection point of the curve agrees with the $\mathrm{pK}_{a 1}$. The tertiary amino groups of most drugs investigated have $\mathrm{pK}_{a}$ values of 7.5 to 8 at physiological salt concentrations (A. Seelig and S. Lotz, unpublished results). Charge repulsion is therefore low at $\mathrm{pH} 8.0$ and the surface area measured corresponds to the true crosssectional area, $A_{D}$, at least to a first approximation. Results in the Table show that for most compounds surface areas measured at $\mathrm{pH} 7.4$ and 8.0 are identical within error limits.

\section{The $\mathrm{K}_{\mathrm{aw}}^{-1} / \mathrm{CMC}_{\mathrm{D}}$ Calibration Diagram}

Figure 3 correlates the $K_{a w}$ values of the drugs measured with the respective $C M C_{D}$ s. The compounds studied previously (Seelig et al., 1994) are indicated as circles and the new compounds as squares. Compounds which easily cross the BBB by passive diffusion $\left(\mathrm{CNS}^{+}\right.$compounds) are represented by open symbols, $\mathrm{CNS}^{-}$compounds by closed symbols. Here $K_{a w}^{-1}$ replaces the 


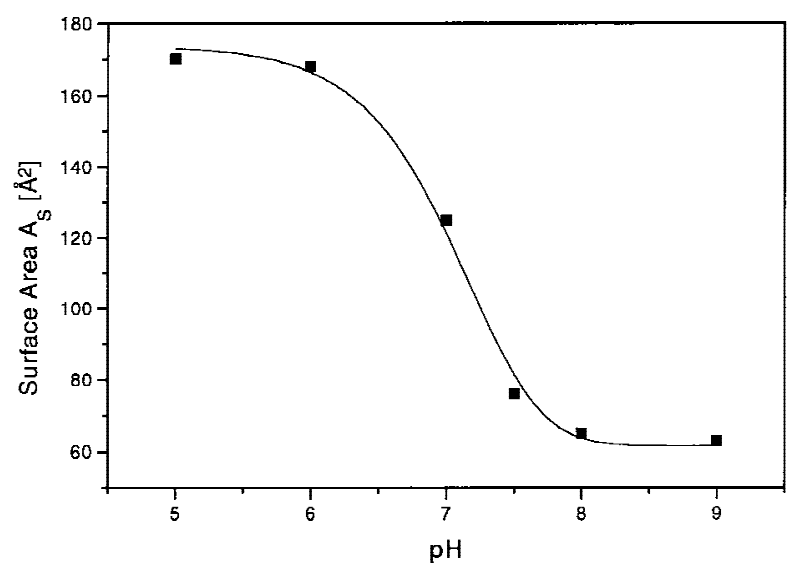

Fig. 2. The surface area, $A_{S}$, of hydroxyzine measured as a function of $\mathrm{pH}$. Buffer solutions (Mes/ $\mathrm{HCl}$ for $\mathrm{pH} 5$ to 7 and $\mathrm{Tris} / \mathrm{HCl}$ for $\mathrm{pH} 7$ to 9) were $50 \mathrm{~mm}$ and contained $114 \mathrm{~mm} \mathrm{NaCl}$. The solid line was calculated using $r_{i}=r_{o}+$ const. $\left(10^{p K_{a}-p H} /\left(1+10^{p K_{a}-p H}\right)\right)^{2}$, where $r_{i}$ is the radius of the cross-sectional area of the ionized form, $r_{o}$, the radius in the absence of charge repulsion, const. a constant and $\mathrm{pK}_{a}$ the $\mathrm{pK}_{a 1}$ of hydroxizine. The inflection point of the curve corresponds to $\mathrm{pK}_{a 1}$. At $\mathrm{pH} 8.0$ charge repulsion effects between molecules at the air-water interface are minimal and therefore the surface area, $A_{s}$, approximately correspond to the cross-sectional area, $A_{D}$, of the molecule.

empirical parameter $C_{0}$ of our earlier work (Seelig et al., 1994). The improved calibration diagram (Fig. 3) essentially agrees with the calibration diagram presented previously (Seelig et al., 1994) and shows three regions which differ with respect to the ability of compounds to cross the BBB by passive diffusion. Region I contains very hydrophobic drugs which fail to cross the BBB. The central region II contains less hydrophobic drugs which cross the BBB easily, with the exception of asimadoline (No. 37) for which passive diffusion is hindered. As will be shown below, this unexpected behavior can be explained by the large cross-sectional area of this molecule. Region III contains hydrophilic drugs which cross the BBB only if applied at high concentrations (30-2400 mg/day). The only $\mathrm{CNS}^{+}$compound applied at distinctly lower concentrations $(\sim 1 \mathrm{mg} /$ day $)$ (Martindale, 1996) is clonidine (No. 19) which has, recently been shown to be transported actively (Huwyler et al., 1997). Three further compounds, salbutamol, sumatriptan, and mequitazine (indicated by half-filled symbols) are also applied at relatively low concentrations (c $\leqslant 16 \mathrm{mg}$ /day) and therefore clinically appear as $\mathrm{CNS}^{-}$ compounds.

\section{The $\mathrm{K}_{\mathrm{aw}}^{-1} / \mathrm{CMC}_{\mathrm{D}} / \mathrm{A}_{\mathrm{D}}$ CALibration Diagram}

During the course of our investigation it became clear that the cross-sectional area of a drug molecule is an important parameter in determining its ability to diffuse through the BBB. This is illustrated by Fig. 4 where the

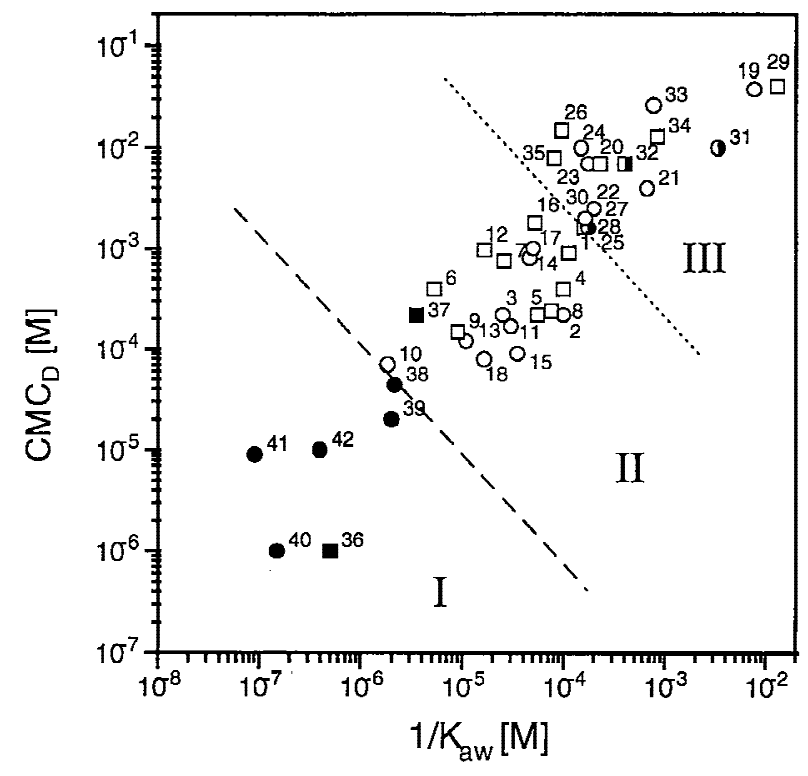

Fig. 3. $K_{a w} / C M C_{D}-$ calibration diagram. The critical micelle concentration, $C M C_{D}$, plotted as a function of the air-water partition coefficient, $K_{a w}^{-1}$, for compounds with known ability to cross the BBB. Region I contains the hydrophobic $\mathrm{CNS}^{-}$compounds, region II the more hydrophilic $\mathrm{CNS}^{+}$compounds, and region III the most hydrophilic compounds which only reach the CNS if applied at relatively high concentrations. The border between region I and II is indicated by a dashed, and the border between region II and III by a dotted line. The two border lines were determined empirically (Seelig et al., 1994). Measurements were performed at $\mathrm{pH} 8.0$ (50 mM Tris/ $\mathrm{HCl}, 114 \mathrm{~mm}$ $\mathrm{NaCl}$ ). The numbers of the data points relate to the Table. Closed symbols $(\boldsymbol{\square}, \mathbf{\square})$ indicate $\mathrm{CNS}^{-}$compounds, open symbols $(\bigcirc, \square)$ $\mathrm{CNS}^{+}$compounds, and half-filled symbols $(\mathbf{O}, \mathbf{\square})$ hydrophilic compounds which are pharmacologically applied at low concentrations and therefore appear as $\mathrm{CNS}^{-}$. Circles $(\mathbf{O}, \bigcirc)$ represent compounds measured previously (Seelig et al., 1994), and squares ( $\square, \mathbf{\square}, \mathbf{\square})$ new compounds.

surface area, $A_{D}$ is plotted as a function of $K_{a w}^{-1}$ and $C M C_{D}$. The hydrophobic $\mathrm{CNS}^{-}$compounds in region I of Fig. 4 have large cross-sectional areas (average $A_{D} \sim$ $105 \AA^{2}$ ) while the more hydrophilic $\mathrm{CNS}^{+}$compounds in regions II and III have average cross-sectional areas of $A_{D} \sim 52 \AA^{2}$ and $A_{D} \sim 41 \AA^{2}$, respectively. The only $\mathrm{CNS}^{-}$compound found in region II (asimadoline) has a cross-sectional area $\left(A_{D}=81 \pm 1 \AA^{2}\right)$ which is distinctly larger than that of the average $\mathrm{CNS}^{+}$compound. This suggests that passive diffusion of compounds with crosssectional areas $A_{D}>80 \AA^{2}$ is strongly reduced.

The importance of cross-sectional areas for passive diffusion is further demonstrated by the following sequence of compounds with similar $K_{a w}$ values but increasing cross-sectional areas: cis-flupentixol $\left(A_{D}=60\right.$ $\left.\AA^{2}, 434.5 \mathrm{Da}\right)$, asimadoline $\left(A_{D}=81 \AA^{2}, 414.5 \mathrm{Da}\right.$, ) and astemizole $\left(A_{D}=94 \AA^{2}, 458.5 \mathrm{Da}\right)$. The compounds show decreasing abilities to reach the brain in the above order. It is interesting to note that molecular mass does not necessarily correlate with the cross-sectional area. 


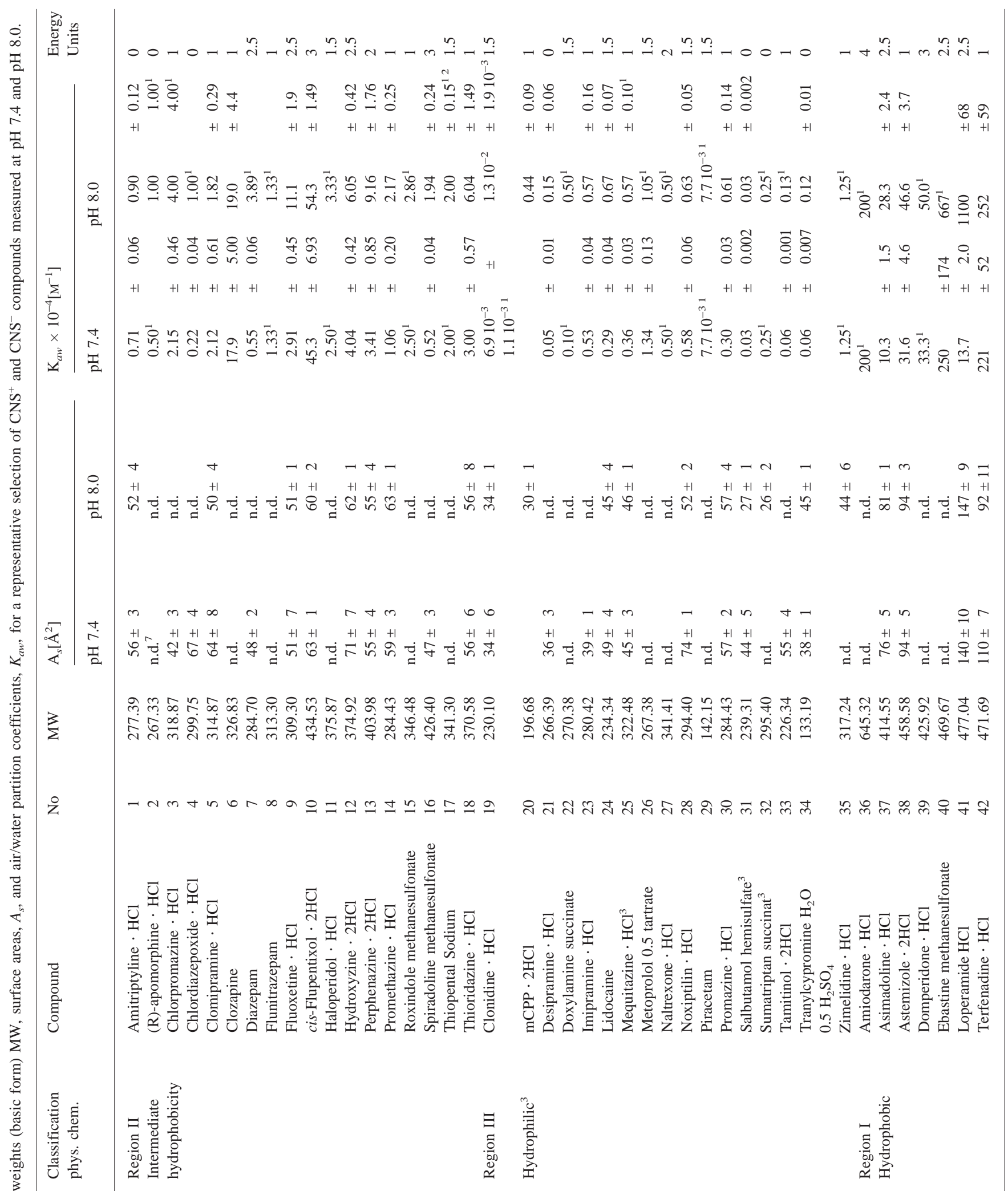



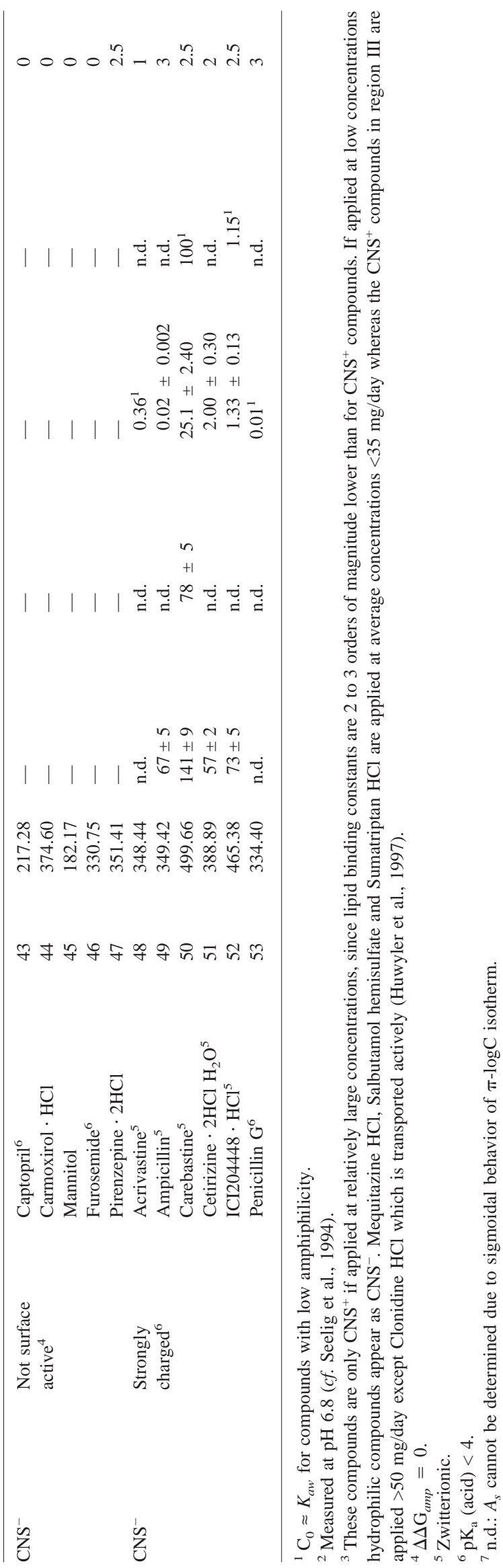

\section{The Influence of Charge on Passive Diffusion Through THE BBB}

The charged substituents of the $\mathrm{CNS}^{+}$compounds shown in the Table are either tertiary amino groups with $\mathrm{pK}_{a}<$ 9 under physiological conditions $(150 \mathrm{~mm} \mathrm{NaCl})$ or a sulfhydryl group (thiopental) with a $\mathrm{pK}_{a}=7.4$. To investigate the role of charge for membrane diffusion we investigated the following compounds with $\mathrm{pK}_{a} \leqslant 4$ : penicillin $\left(\mathrm{pK}_{a 1}=2.8\right)$, cetirizine $\left(\mathrm{pK}_{a 1}=3.7, \mathrm{pK}_{a 2}=\right.$ 8.2), ampicillin $\left(\mathrm{pK}_{a 1}=3.0, \mathrm{pK}_{a 2}=7.1\right)$, acrivastine $\left(\mathrm{pK}_{a 1}=2.8, \mathrm{pK}_{a 2}=7.5\right)$, ICI204448 $\left(\mathrm{pK}_{a 1}=3.1\right.$, $\mathrm{pK}_{a 2}=8.0$ ). The $\mathrm{pK}_{a}$ values of the first two compounds have been determined experimentally (ter Laak et al., 1994; Tsuji et al., 1977), the others were estimated according to the method of Perrin, Dempsey \& Serjeant (1981). Measurement of the Gibbs adsorption isotherm shows that these compounds are all amphiphilic and are located in regions II and III ( $c f$. Table, not included in Fig. 3) and are thus predicted to cross the BBB. Nevertheless these compounds are not found to penetrate into the brain. We therefore conclude that compounds with minimally one charge with a $\mathrm{pK}_{a}<4$ for acids and correspondingly a $\mathrm{pK}_{a}>10$ for bases do not cross the BBB by passive diffusion.

\section{Discussion}

53 compounds with known propensity to cross the BBB were characterized with surface activity measurements. Three parameters, the air-water partition coefficient, $K_{a w}$, the critical micelle concentration, $C M C_{D}$, and the cross-sectional area, $A_{D}$ were deduced. Figure 3 demonstrates that a $K_{a w}^{-1}$ vs. $C M C_{D}$ diagram provides a useful means to classify these drugs as $\mathrm{CNS}^{-}$and $\mathrm{CNS}^{+}$. Most notable is region I which comprises a number of drugs which are very hydrophobic according to most classical criteria but still cannot pass the BBB. Inspection of Fig. 4 shows that these drugs are characterized by crosssectional areas $A_{D} \geqslant 80 \AA^{2}$. Combining this finding with the specific structure of the lipid bilayer provides a clue for their inability to cross the BBB by passive diffusion.

The Role of the Molecular Cross-Sectional Area FOR PARTITIONING INTO THE LIPID MEMBRANE

Boguslavsky et al. (1994) have shown that the penetration of a molecule between the fatty acyl chains of a lipid monolayer requires work, $\Delta W$ which is proportional to the surface pressure, $\pi_{m}$, of the monolayer and the crosssectional area, $A_{D}$, of the penetrating compound

$\Delta W=A_{D} \pi_{m}$

The partition coefficient for the lipid monolayer is thus proportional to $\exp \left(-A_{D} \pi_{m} / k T\right)$. For a lipid bilayer the situation is similar. A penetrating substance has to per- 


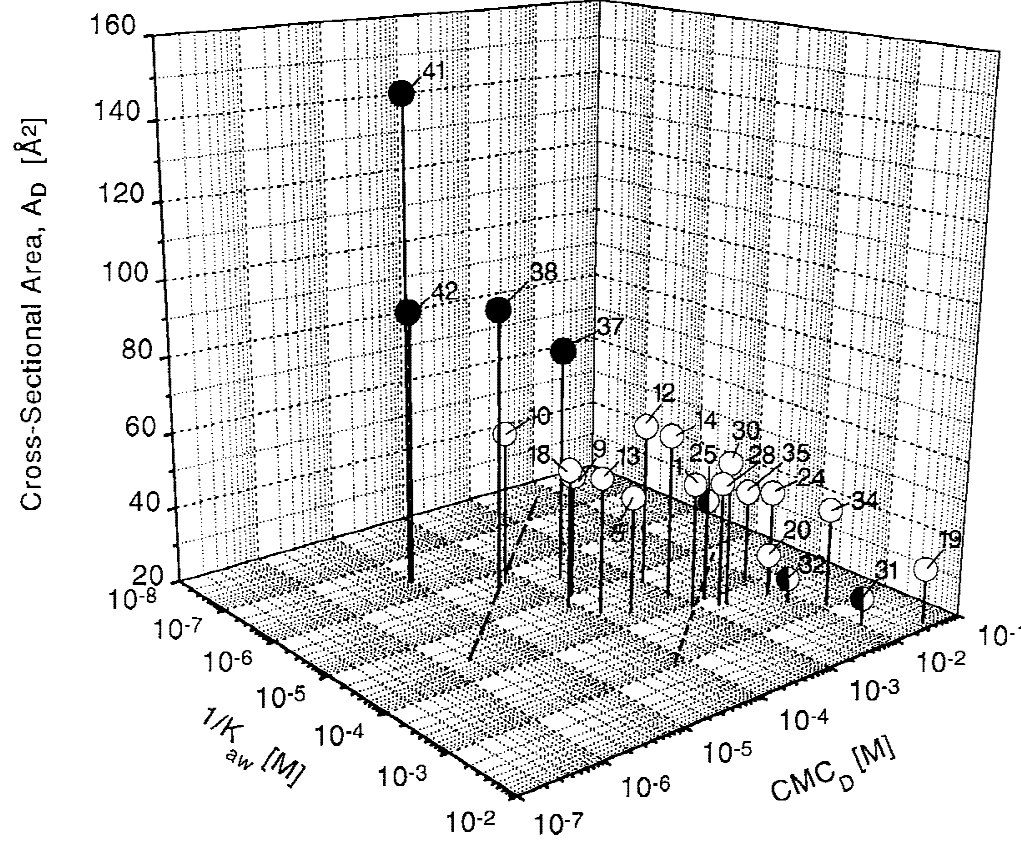

Fig. 4. $K_{a w} / C M C_{D} / A_{D}-$ calibration diagram measured at $\mathrm{pH} 8.0(50 \mathrm{~mm}$ Tris/ $\mathrm{HCl}, 114 \mathrm{~mm}$ $\mathrm{NaCl}$ ). The air-water partition coefficient, $K_{a w}^{-1}$ is plotted in the $x$-, the critical micelle concentration, $C M C_{D}$, in the $y$ - and the cross-sectional area, $A_{D}$ in the $z$-axes. For symbols $c f$. legend to Fig. 3. Compounds with low amphiphilicity or amphiphilicity changing in the course of aggregation are not included. form work against the internal lateral pressure of the lipid bilayer, $\pi_{b i}^{2}$

$\Delta W=A_{D} \pi_{b i}$

The lipid-water partition coefficient, $K_{l w}$, is thus given by

$K_{l w}=K_{O} \exp \left(-A_{D} \pi_{b i} / k T\right)$

where $K_{O}$ is a proportionality constant. Since the airwater interface resembles the lipid-water interface with respect to the gradient of dielectric constants, $\varepsilon$ ( $\varepsilon \cong 1$ for air, $\varepsilon \cong 2$ for the interior of the lipid membrane, and $\varepsilon \cong$ 80 for water) and since the surface pressure of the drug monolayer is negligible at the concentration, $1 / K_{a w}$, we assume that $K_{O}$ is proportional to $K_{a w}$. This assumption was tested with a series of compounds for which both $K_{l w}$ and $K_{a w}$ are known. The lipid-water partition coefficients, $K_{l w}$, were measured for small unilamellar 1palmitoyl-2-oleoyl-sn-phosphocholine (POPC) vesicles

\footnotetext{
${ }^{2}$ The effective internal lateral pressure in phospholipid bilayers was previously estimated in comparison to lipid monolayers. A requirement for the correspondence between the two is that the area per lipid molecule in the monolayer and the bilayer should be the same. Experimentally the best correspondence was obtained for monolayers at a surface pressure of 30-35 mN/m (for review see Marsh, 1996). A somewhat wider surface pressure range $(\sim 28-40 \mathrm{mN} / \mathrm{m})$ is discussed by Macdonald (1996).
}

with high sensitivity titration calorimetry (A. Frentzel, X. $\mathrm{Li}$, and A. Seelig; in preparation). Figure 5 shows a plot of $\ln \left(K_{l w} / K_{a w}\right) v s . A_{D}$. A linear regression analysis of the experimental data yields a straight line with a slope of $\pi / k T=0.083 \pm 0.014\left[\AA^{-2}\right]$. The internal lateral pressure of the lipid bilayer ${ }^{3}$ is calculated as $\pi_{b i}=34 \pm 4$ $\mathrm{mN} / \mathrm{m}$ which is in agreement with the monolayer-bilayer (POPC) equivalence pressure, $\pi=32 \pm 1 \mathrm{mN} / \mathrm{m}$ determined earlier (Seelig, 1987).

An exponential decrease of the lipid-water partition coefficients with increasing cross-sectional areas is supported by earlier measurements showing an exponential decrease of binding (Gobas et al., 1988) to -, and permeation through (Lieb \& Stein, 1986) lipid membranes with increasing molecular volume.

At present the lateral packing density of the BBB is not known, but an estimation is possible on the basis of its cholesterol content. The content of cholesterol expressed as mole \% of total lipids of endothelial membranes amounts to $\sim 30 \%$ (Simons \& van Meer, 1988). The cholesterol/phospholipid ratio of cerebral microvessels has been determined as 0.7 (mole/mole) (Mooradian \& Meredith, 1992) in close agreement with that of other endothelial cells (Simons \& van Meer, 1988). The effect of cholesterol is to increase the order parameter of the membranes in the liquid crystalline phase (Gally, Seelig $\&$ Seelig, 1976). Upon addition of 30-40\% cholesterol to POPC membranes the lateral packing density has been

\footnotetext{
${ }^{3}$ For low drug concentrations in the lipid membrane the lateral packing density is assumed to remain constant to a first approximation.
} 


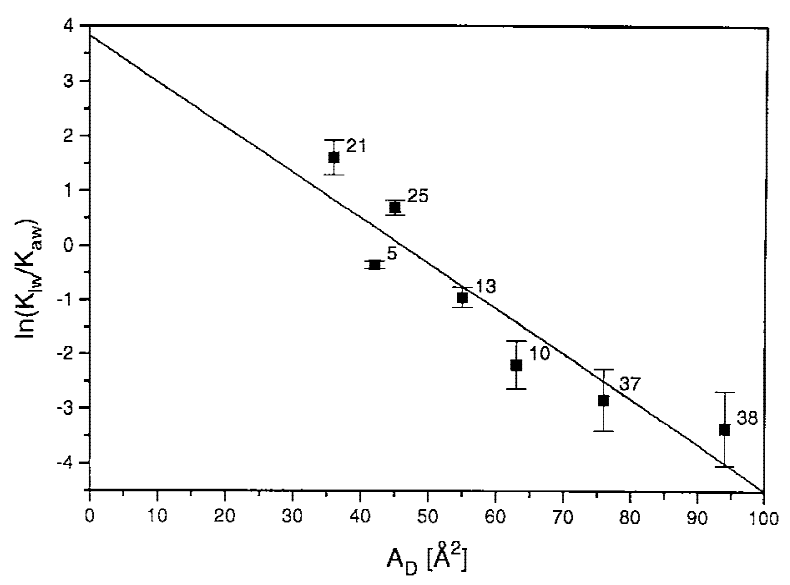

Fig. 5. The natural logarithm of the quotient of the lipid membranewater partition coefficient, $K_{l w}$, and the air-water partition coefficient, $K_{a w}$, plotted as a function of the cross-sectional area. A linear regression analysis yields a straight line with a slope of $\pi_{b i} / k T=0.084 \pm$ $0.015\left[\AA^{-2}\right]$ and the internal lateral bilayer pressure is calculated as $\pi_{b i}$ $=34 \pm 4 \mathrm{mN} / \mathrm{m}$. The compounds measured are: chlorpromazine $\mathrm{HCl}$ (3), cis-flupentixol $2 \mathrm{HCl}(10)$, desipramine $\mathrm{HCl}(21)$, mequitazine $\mathrm{HCl}$ (25), asimadoline $\mathrm{HCl}$ (37), and astemizole $2 \mathrm{HCl}$ (38). All parameters were measured at $\mathrm{pH} 7.4(50 \mathrm{~mm}$ Tris/ $\mathrm{HCl}, 114 \mathrm{~mm} \mathrm{NaCl})$, with the exception of $K_{l w}$ for astemizole and asimadoline which were measured at $\mathrm{pH} 5.2$ due to solubility problems. Electrostatic effects were taken into account by means of the Gouy-Chapman theory.

shown to shift from $32 \mathrm{mN} / \mathrm{m}$ (Seelig, 1987) to $35 \mathrm{mN} / \mathrm{m}$ (Demel et al., 1975; Taschner, 1993).

For a membrane with a lateral packing density of $\pi$ $=35 \mathrm{mN} / \mathrm{m}$ increasing the cross-sectional area of a molecule with a given partition coefficient, $K_{a w}$, from $A_{D}=$ $52 \AA^{2}$ (average area in region II) to $105 \AA^{2}$ (average area in region I) decreases the partition coefficient, $K_{l w}$ on average by a factor of $\sim 100$. The limiting cross-sectional area for passive diffusion through the BBB obtained from the calibration diagram (Fig. 5) is $\sim 80 \AA^{2}$. For a given $K_{a w}$ an increase of the cross-section from $52 \AA^{2}$ to $80 \AA^{2}$ reduces the $K_{l w}$ by a factor of $\sim 10$.

Quite in contrast to the partitioning into lipid membranes, partitioning into isotropic solvents like hexane or octanol has been shown to increase with the molecular volume of the compound (Gobas et al., 1988). Predictions of passive diffusion through the BBB based on partition coefficient measurements between water and either isotropic solvents or loosely packed anisotropic systems with low lateral packing densities $(\pi<32$ $\mathrm{mN} / \mathrm{m}$ ) will therefore be misleading for molecules with large cross-sectional areas.

\section{The Role of Amphiphilicity AND ChaRge For Membrane Diffusion}

Amphiphilic molecules dissolved in water can achieve segregation of their hydrophobic portion from the sol- vent either by partitioning into the air-water interface or by self-association. While both processes are primarily entropy driven (hydrophobic effect), micelle formation requires the additional action of an opposing force. In the case of charged head groups it arises from electrostatic repulsion and in the case of hydrophilic head groups from the preference for hydration ( $c f$. Tanford, 1987). Figure $1 A$ shows that $K_{a w}<C M C$. The free energy of micelle formation $\Delta G_{\text {mic }}=R T \ln [C M C]$ (Tausk et al., 1974) is therefore smaller than the free energy of partitioning into the air-water interface, $\Delta G_{a w}=-R T$ $\ln K_{a w}$. The difference between the free energy of transfer of a compound from the aqueous phase to the airwater interface, and the free energy of micelle formation directly reflects the amphiphilicity of a compound. We therefore define amphiphilicity as

$$
\Delta \Delta G_{a m}=\Delta G_{a w}-\Delta G_{m i c}
$$

At constant charge amphiphilicity, $\Delta \Delta \mathrm{G}_{a m}$, is proportional to the distance between the charged residue and the most remote hydrophobic residue, e.g., for fatty acids the amphiphilicity increases by $-1.14 \pm 0.21 \mathrm{~kJ} /$ mole per $-\mathrm{CH}_{2}$ group. The amphiphilicity observed for the drugs investigated is in the range of $\Delta \Delta G_{a m}=-3$ to -11 $\mathrm{kJ} / \mathrm{mol}$. Most commonly amphiphilicity is around -5.6 $\mathrm{kJ} / \mathrm{mol}$ (molecules on the diagonal between the lower left and the upper right corner in Figure 3). Compounds which lack amphiphilicity $\left(\Delta \Delta G_{a m} \cong 0\right)$ such as e.g., carmoxirol and pirenzepine are not surface active and are thus unable to insert into a lipid membrane. ${ }^{4}$

Although amphiphilicity is a prerequisite for membrane binding it is, per se, not sufficient to allow diffusion through a membrane. For membrane permeation a loss of charge, observed as a $\mathrm{pK}_{a}$ shift upon membrane binding (Beschiaschvili \& Seelig, 1992) or a delocalization of charge e.g., in the tetraphenyl phosphonium (Altenbach \& Seelig, 1985) or tetraphenyl borate ion (Seelig $\&$ Ganz, 1991) is required. As seen in the Table compounds which carry at least one stable charge $\mathrm{pK}_{a}<4$ for acids or $\mathrm{pK}_{a}>10$ (no example shown) do not cross the BBB.

In summary, we have shown that the drug partition coefficient for lipid membranes, $K_{l w}$, can be quantitatively predicted on the basis of the air-water partition coefficient, $K_{a w}$, and the cross-sectional area of the molecule, $A_{D}$, taking into account the internal lateral bilayer pressure. We have further shown that permeation of a drug molecule through the lipid membrane of the BBB is

\footnotetext{
${ }^{4}$ Hydrophobic drugs with a low amphiphilicity tend to associate in solution and can therefore not insert as monomers into membranes. This is different for small molecules such as oxygen which dissolve in aqueous solution and enter the membrane despite the lack of amphiphilicity.
} 
optimal if (i) amphiphilicity is $\Delta \Delta G_{a m}>-3 \mathrm{~kJ} / \mathrm{mole}$, (ii) the $K_{a w}$ value is in the range of $10^{5}-10^{3} \mathrm{M}^{-1}$, (iii) the cross-sectional area is $A_{D}<80 \AA^{2}$ and (iv) the ionization constants are $\mathrm{pK}_{a}>4$ for acids and $\mathrm{pK}_{a}<10$ for bases. The present method is suited as a fast screening method and is superior in its predictive power to a single parameter organic phase/water partition equilibrium measurement.

We thank Sandra Lotz and Thomas Alt for expert technical assistance and Dr. Heinrich Stahl for helpful discussions. This research was supported by the Swiss National Science Foundation Grant 31.42058.94

\section{References}

Altenbach, C., Seelig, J. 1985. Binding of the lipophilic cation tetraphenylphosphonium to phosphatidylcholine membranes. Biochim. Biophys. Acta 818:410-415

Barber, A., Bartoszyk, G.D., Bender, H.M., Gottschlich, R., Greiner, H.E., Harting, J., Mauler, F., Minck, K.O., Murray, R.D., Simon, M. 1994. A pharmacological profile of the novel, peripherallyselective kappa- opioid receptor agonist, EMD 61753. Br. J. Pharmacol. 113:1317-1327

Bassolino-Klimas, D., Alper, H.E., Stouch, T.R. 1993. Solute diffusion in lipid bilayer membranes: an atomic level study by molecular dynamics simulation. Biochemistry 32:12624-12637

Beschiaschvili, G., Seelig, J. 1992. Peptide binding to lipid bilayers. Nonclassical hydrophobic effect and membrane-induced $\mathrm{pK}$ shifts. Biochemistry 31:10044-10053

Boguslavsky, V., Rebecchi, M., Morris, A.J., Jhon, D.Y., Rhee, S.G., McLaughlin, S. 1994. Effect of monolayer surface pressure on the activities of phosphoinositide-specific phospholipase C-beta 1, -gamma 1, and -delta 1. Biochemistry 33:3032-3037

Broekhuysen, J., Laruel, R., Sion, R. 1969. [Research on the benzofuran series. XXXVII. Comparative study of transit and metabolism of amiodarone in different species of animals and humans]. Arch. Int. Pharmacodyn. Ther. 177:340-359

Büldt, G., Gally, H.U., Seelig, A., Seelig, J., Zaccai, G. 1978. Neutron diffraction studies on selectively deuterated phospholipid bilayers. Nature 271:182-184

Cevc, G., Marsh, D. 1987. Phospholipid Bilayers, Physical Principles and Models. Wiley, New York

Demel, R.A., Geurts van Kessel, W.S., Zwaal, R.F., Roelofsen, B., van Deenen, L.L. 1975. Relation between various phospholipase actions on human red cell membranes and the interfacial phospholipid pressure in monolayers. Biochim. Biophys. Acta 406:97-107

Fischer, H. 1998. Ph.D. Thesis University of Basel

Fuller, R.W. 1986. Substituted phenylpiperazines as serotonin agonists: structural determinants of potency and interaction with receptor subtypes. Psychopharmacol. Bull. 22:825-828

Gally, H.U., Seelig, A., Seelig, J. 1976. Cholesterol-induced rod-like motion of fatty acyl chains in lipid bilayers a deuterium magnetic resonance study. Hoppe Seylers Z. Physiol. Chem. 357:1447-1450

Gobas, F.A., Lahittete, J.M., Garofalo, G., Shiu, W.Y., Mackay, D. 1988. A novel method for measuring membrane-water partition coefficients of hydrophobic organic chemicals: comparison with 1-octanol-water partitioning. J. Pharm. Sci. 77:265-272

Heykants, J., Michiels, M., Knaeps, A., Brugmans, J. 1974. Loperamide (R 18 553), a novel type of antidiarrheal agent. Part 5: the pharmacokinetics of loperamide in rats and man. Arzneimittelforschung 24:1649-1653

Huwyler, J., Fricker, G., Torok, M., Schneider, M., Drewe, J. 1997.
Transport of clonidine across cultured brain microvessel endothelial cells. J. Pharmacol. Exp. Ther. 282:81-85

Kaliszan, R. 1990. High Performance Liquid Chromatographic Methods and Procedures of Hydrophobicity Determination. Quant. Struct.-Act. Relat. 9:83-87

Leeson, G.A., Chan, K.Y., Knapp, W.C., Biedenbach, S.A., Wright, G.J., Okerholm, R.A. 1982. Metabolic disposition of terfenadine in laboratory animals. Drug Res. 32:1173-1178

Lieb, W.R., Stein, W.D. 1986. Non-Stokesian nature of transverse diffusion within human red cell membranes. J. Membrane Biol. 92:111-119

Macdonald, R.C. 1996. The relationship and interactions between lipid bilayers vesicles and lipid monolayers at the air/water interface. Marcel Dekker, New York, Basel, Hong Kong

Marsh, D. 1996. Lateral pressure in membranes. Biochim. Biophys. Acta 1286: $183-223$

Martindale, N.O. 1996. The Extra Pharmacopoeia. The Royal Pharmaceutical Society, London Martinez-Tobed, A., Tarrus, E., Segura, J., Roberts, D.J. 1992. Pharmacokinetic studies of ebastine in rats, dogs and man. Drugs of Today 28:57-67

Matsuda, M., Sakashita, M., Mizuki, Y., Yamaguchi, T., Fuji, T., Sekine, Y. 1994. Comparative pharmacokinetiks of the histamine $\mathrm{H}_{1}$-receptor antagonist ebastine and its active metabolite carebastine in rats, guinea pigs, dogs and monkeys. Drug Res. 44:55-59

Mayer, L.D., Wong, K.F., Menon, K., Chong, C., Harrigan, P.R., Cullis, P.R. 1988. Influence of ion gradients on the transbilayer distribution of dibucaine in large unilamellar vesicles. Biochemistry 27:2053-2060

McLaughlin, S. 1989. The electrostatic properties of membranes. Annи. Rev. Biophys. Biophys. Chem. 18:113-136

Mooradian, A.D., Meredith, K.E. 1992. The effect of age on protein composition of rat cerebral microvessels. Neurochem. Res. 17:665670

Moragues, J., Roberts, D.J. 1990. Ebastine. Drug Fut. 15:674-679

Newton, W., Kluza, R.B. 1978. $\mathrm{pK}_{\mathrm{a}}$ values of medicinal compounds in pharmacy practice. Drug Intelligence \& Clinical Pharmacy 12:546-554

Ong, S., Liu, H., Qui, X., Bhat, G., Pidgeon, C. 1995. Membrane partition coefficients chromatographically measured using immobilized artificial membrane surfaces. Anal. Chem. 67:755-762

Paula, S., Süs, W., Tuchtenhagen, J., Blume, A. 1995. Thermodynamics of Micelle Formation as a Function of Temperature: A High Sensitivity Titration Calorimetry Study. J. Phys. Chem. 99:1174211751

Perrin, D.D., Dempsey, B., Serjeant, E.P. 1981. pKa Prediction for Organic Acids and Bases. Chapman and Hall, London and New York

Reece, P.A., Sedman, A.J., Rose, S., Wright, D.S., Dawkins, R., Rajagopalan, R. 1994. Diuretic effects, pharmacokinetics, and safety of a new centrally acting kappa-opioid agonist (CI-977) in humans. J. Clin. Pharmacol. 34:1126-1132

Reyntjens, A.J., Niemegeers, C.J., Van Neuten, J.M., Laduron, P., Heykants, J., Schellekens, K.H., Marsboom, R., Jageneau, A., Broekaert, A., Janssen, P.A. 1978. Domperidone, a novel and safe gastrokinetic anti-nauseant for the treatment of dyspepsia and vomiting. Arzneimittelforschung 28:1194-1196

Rosen, M.J. 1989. Surfactants and Interfacial Phenomena. John Wiley \& Sons, New York

Schuermans, V., Van Lommel, R., Dom, J., Brugmans, J. 1974. Loperamide (R18553), a novel type of antidiarrheal agent. Arzneim. Forsch. 24:1653-1657

Seelig, A. 1987. Local anesthetics and pressure: a comparison of dibucaine binding to lipid monolayers and bilayers. Biochim. Biophys. Acta 899:196-204 
Seelig, A. 1998. A general pattern for substrate recognition by Pglycoprotein. Eur. J. Biochem. 251:252-261

Seelig, J., Ganz, P. 1991. Nonclassical hydrophobic effect in membrane binding equilibria. Biochemistry 30:9354-359

Seelig, A., Gottschlich, R., Devant, R.M. 1994. A method to determine the ability of drugs to diffuse through the blood-brain barrier. Proc. Natl. Acad. Sci. USA 91:68-72

Seelig, J., Seelig, A. 1974. Deuterium magnetic resonance studies of phospholipid bilayers. Biochem. Biophys. Res. Commun. 57:406411

Seelig, J., Seelig, A. 1980. Lipid conformation in model membranes and biological membranes. Q. Rev. Biophys. 13:19-61

Seppala, T., Savolainen. 1982. Effect of astemizole on human psychomotor performance. Curr. Ther. Res. 31:638-644

Simons, K., van Meer, G. 1988. Lipid sorting in epithelial cells. Biochemistry 27:6197-6202

Tanford, C. 1987. Amphiphile orientation: physical chemistry and biological function. Biochem. Soc. Trans. 15 Suppl:1S-7S

Taschner, N. 1993. Diploma Thesis University of Basel

Tausk, R.J., Karmiggelt, J., Oudshoorn, C., Overbeek, J.T. 1974. Physi- cal chemical studies of short-chain lecithin homologues. I. Influence of the chain length of the fatty acid ester and of electrolytes on the critical micelle concentration. Biophys. Chem. 1:175-183

ter Laak, A.M., Tsia, R.S., Donne-Op den Kelder, G.M., Carrupt, P.-A., Testa, B., Timmerman, H. 1994. Lipophilicity and hydrogenbonding capacity of $\mathrm{H} 1$-antihistaminic agents in relation to their central sedative side-effects. Eur. J. Pharm. Sci. 2:373-384

Tsuji, A., Kubo, O., Miyamoto, E., Yamana, T. 1977. Physicochemical properties of beta-lactam antibiotics: oil-water distribution. $J$. Pharm. Sci. 66:1675-1679

Von Voightlander, P.F., Lewis, R.A. 1988. Analgesic and mechanistic evaluation of spiradoline, a kappa opioid. J. Pharmacol. Exp. Ther. 246:259-262

Wenk, M.R., Alt, T., Seelig, A., Seelig, J. 1997. Octyl-beta-Dglucopyranoside partitioning into lipid bilayers: thermodynamics of binding and structural changes of the bilayer. Biophys. J. 72:17191731

Yang, C.Y., Cai, S.J., Liu, H., Pidgeon, C. 1997. Immobilized artificial membranes-screens for drug membrane interactions. Adv. Drug Delivery Rev. 23:229-256 